\title{
Power-Aware Scheduling and Dynamic Voltage Setting for Tasks Running on a Hard Real-Time System ${ }^{1}$
}

\author{
Peng Rong \\ Dept. of Electrical Engineering \\ University of Southern California \\ Los Angeles, CA 90089 \\ e-mail : prong@usc.edu
}

\author{
Massoud Pedram \\ Dept. of Electrical Engineering \\ University of Southern California \\ Los Angeles, CA 90089 \\ e-mail : pedram@ceng.usc.edu
}

\begin{abstract}
This paper addresses the problem of minimizing energy consumption of a computer system performing periodic hard real-time tasks with precedence constraints. In the proposed approach, dynamic power management and voltage scaling techniques are combined to reduce the energy consumption of the CPU and devices. The optimization problem is first formulated as an integer programming problem. Next, a three-phase solution framework, which integrates power management scheduling and task voltage assignment, is proposed. Experimental results show that the proposed approach outperforms existing methods by an average of $18 \%$ in terms of the system-wide energy savings.
\end{abstract}

\section{INTRODUCTION}

Reducing power consumption is a key requirement for extending the battery service lifetime of portable devices. Even in high-end computer systems, expensive cooling and packaging cost and declined reliability associated with high levels of power dissipation, make low power design a critical design consideration. Dynamic power management (DPM) and dynamic voltage scaling (DVS) have both proven to be highly effective techniques for reducing power dissipation in such systems. DPM refers to a selective shut-off of idle system components, while DVS slows down underutilized resources and decreases their operating voltages. A detailed survey of DPM techniques can be found in [1].

Most researches on low-power task scheduling focus only on reducing the CPU power by using DVS techniques. However, in reality, executing a useful task on a computer system requires cooperation between the CPU and many other system components, e.g., memory, disk drives, wireless devices, etc., which can also consume significant amounts of power. These components generally have their own voltage levels and may or may not support DVS, which makes it difficult to apply DVS techniques to the CPU only and achieve total system power savings. In fact, DVS when applied to CPU only may even increase the overall system energy consumption for executing a given set of tasks. At the same time, DPM is known to be an effective approach for reducing the power consumption of the various peripheral components and I/O devices. Thus DVS combined with DPM has the potential to achieve power savings, not possible by either DPM or DVS.

\footnotetext{
${ }^{1}$ This project was funded in part by the NSF CNS grant no. 0509564.
}

This paper addresses the problem of power optimization of a real-time system having heterogeneous components and performing periodic hard real-time tasks. The dependencies between the tasks are described by a directed acyclic graph (DAG), sometimes referred to as a task graph.

Most related work on low power scheduling for dependent tasks concentrate on DVS techniques. Some authors have considered voltage assignment on distributed embedded systems. The approach proposed in [2] first schedules tasks based on a list-scheduling algorithm by using the reciprocal of the slack time as the task priority, and next tries to evenly distribute the available positive slack time among tasks on each critical path and thereby reduce the operating voltages and save energy. Reference [3] assumes a given task schedule and assignment and proposes an extended list-scheduling algorithm. At each time step, the energy saving of a task is calculated as the difference between the expected energies given the task is scheduled at this step or at the next step. A task with a higher energy saving and less slack time has a higher priority. The authors of [4] present a two-phase framework. In the first phase, a version of the early-deadlinefirst scheduling is used to assign a task to a best-fit processor in terms of the task ready time and the processor free time. In the second phase, an ILP optimization problem is formulated and solved in order to determine the voltage level of the processor used to run each task.

Several works on DPM-based task scheduling have also been proposed in the literature. An online scheduling algorithm for independent tasks is presented in [5]. This algorithm attempts to reduce the number of device on/off transitions by greedily extending the pattern for current device usage so as to reduce average power consumption in the near future. Reference [6] proposes an offline branch-and-bound algorithm to search for the energy optimal task scheduling. In [7], the authors prove that solving energy optimal task scheduling for DPM on multiple devices is an NP hard problem even for a simple case where no timing dependency is considered. References [8] and [9] start with a given timing-fixed task sequence and propose algorithms to determine an energy-minimal state transition sequence for devices while satisfying hard time constraints.

More recently, a number of researchers have reported DVS algorithms taking into account energy consumption of 
the system components. In [10] the authors present a DVS heuristic based on the critical speed of each task, which is defined as the CPU speed at which the execution of a task consumes the least total system energy. Reference [11] proposes a DVS technique based on a precise energy model considering both the active power and standby component of the system power.

In the literature, several works have been proposed on combining DVS and DPM. Reference [12] present a Markovian decision processes based DPM model which is a uniform modeling framework for both DVS and DPM. In [13], the authors combine DVS with their previously proposed renewal theory based DPM approach. These two stochastic approaches are unable to handle tasks with hardtime constraints or dependency. The problem of combining DVS and DPM for hard real-time tasks is studied in reference [14], where a scheduling algorithm for a single processor with a sleep state is presented which is proved having a competitive ratio of 3 . Task dependency is not considered in this work either.

To the best of our knowledge, no proposed research work is conducted to combine DVS and DPM techniques for hard real-time dependent tasks running on multiple devices. This is specifically the contribution of the present paper. In particular, an integer programming based formulation is first provided to exactly state the optimization problem to be addressed. Next, a three-phase algorithm is proposed to solve the power-aware task scheduling and voltage-to-task assignment problems with the objective of minimizing the total system energy consumption. The three steps are power-aware task scheduling, task-level voltage assignment, and task rescheduling and voltage level refinement.

The remainder of the paper is organized as follows: The problem formulation is presented in section 2 . The three steps of the proposed algorithm are described in sections 3, 4 and 5, respectively. Experimental results and conclusions are given in sections 6 and 7.

\section{Problem Formulation}

This paper targets a real-time system which has a single CPU and $\kappa$ system devices (e.g., various I/O devices, main memory.) The CPU is considered to be device number 0 whereas other devices are numbered from 1 to $\kappa$. The CPU has a discrete number of performance states corresponding to different supply voltage levels and clock frequencies and one sleep state. All other devices have a functional state during which they provide service and a low power sleep mode during which they cannot provide any services. ${ }^{2}$ Furthermore, a device which is in the performance/functional state can be in one of two sub-states: 1) actively performing services; 2) waiting for service requests to arrive. We will refer to substate 1 as the active state and sub-state 2 as the idle state. We assume that each device $k$ consumes the same amount of power when they are in active or idle mode (denoted by

\footnotetext{
${ }^{2}$ It is straight-forward to extend the mathematical formulation to handle I/O devices with multiple low-power states (e.g., standby, drowsy, and sleep.)
}

funcpow $w_{k}$ ), but significantly less power when it is in the sleep mode $\left(\right.$ sleepow $_{k}$.)

A set of $n$ non-preemptive dependent tasks periodically run on the system with a time period $T_{d}$. The data dependency (precedence) constraints between the tasks are described by a directed acyclic task graph, called a task graph, $G(V, E)$, where each node $v$ denotes a task and a directed edge $e(u, v)$ represents a data flow between task $u$ and $v$ and implies that task $v$ can be executed only after task $u$ finishes. Every task has to be performed on the CPU, and may require support (services) from some (or all) of the system devices. It is assumed that during the run time of a task, all devices whose services are required by the task in question will stay in their active modes. The problem is to solve the optimal task scheduling and task-level voltage assignment with the objective of minimizing the total system energy consumption during period $T_{d}$.

Let $V_{i}, i=1, \ldots, m$, denote the $m$ operating voltages for the CPU and $f_{i}$ the clock frequency of the CPU at voltage $V_{i}$. We define the workload of task $u$ as the number of CPU cycles without considering memory and IO device access delay. Let $N_{u, i}$ denote the actual number of CPU cycles required to complete task $u$ at operating voltage $V_{i}$. We define variable $x(u, i)$ to represent the percentage of the workload of task $u$ which is performed at voltage $V_{i}$. Note that there are $m \cdot n$ such variables. The execution time (duration) of task $u$ is calculated as

$$
d u r_{u}=\sum_{i=1}^{m} \frac{x(u, i) \cdot N_{u, i}}{f_{i}}
$$

where $\sum_{i=1}^{m} x(u, i)=1$.

We introduce $n . \kappa$ 0-1 integer variables, $Z_{k}(u)$, as follows: $Z_{k}(u)=1$ exactly if task $u$ requires service from device $k$. The energy consumption due to execution of task $u$ is equal to

$$
\text { ene }_{u}=c_{u} \sum_{i=1}^{m} x(u, i) \cdot N_{u, i} \cdot V_{i}^{2}+\sum_{k=1}^{K} Z_{k}(u) \cdot P_{k} \cdot d u r_{u},
$$

where $c_{u}$ is the effective switched capacitance per CPU cycle; and $P_{k}$ is the power consumption of device $k$ in the active mode.

Let $s(u)$ denote the start time of task $u$. Thus the precedence constraint is expressed as

$$
s(u)+d u r_{u} \leq s(v) \quad \forall e(u, v) \in E
$$

To formulate the energy consumed by the CPU and devices during idle time, we need to introduce two virtual (dummy) tasks: task 0 of duration zero which is placed at exactly the start of period $T_{d}$ and task $n+1$ of duration zero which is placed at the end of period $T_{d}$. We define tasks 0 and $\mathrm{n}+1$ so as to require all devices, i.e., $Z_{k}(0)=1$ and $Z_{k}(n+1)=1, \forall k$. Notice that the interval between task 0 and the first task executed on device $k$ denotes the first idle period. Similarly, the last idle period is defined as the interval between the last task executed on device $k$ and task $n+1$. Also notice that.

We introduce $(n+2)^{2} k 0-1$ integer scheduling variables $Y_{k}(u, v)$ as follows: $Y_{k}(u, v)=1$ exactly if task $u$ is executed on 
device $k$ immediately before task $v$ is executed on the same device. Since on each device, every task has only one immediate successor, the following constraint on $Y_{k}(u, v)$ should be respected

$$
\sum_{v=1}^{n+1} Y_{k}(u, v)=\left\{\begin{array}{ll}
1, & Z_{k}(u)=1 \\
0, & \text { otherwise }
\end{array}, \quad u=0,1, \ldots, n\right.
$$

Similarly, every task has only one immediate predecessor; i.e.,

$$
\sum_{u=0}^{n} Y_{k}(u, v)=\left\{\begin{array}{ll}
1, & Z_{k}(v)=1 \\
0, & \text { otherwise }
\end{array}, \quad v=1,2, \ldots, n+1\right.
$$

There is also a precedence constraint between task $v$ and its immediate successor, both of which are executed on device $k$, as follows

$$
\sum_{u=0}^{n}\left(s(u)+d u r_{u}\right) \cdot Y_{k}(u, v) \leq s(v) \quad \forall v \in V, k \in \operatorname{devs_{v}}
$$

With variable $Y_{k}(u, v)$, we can express the duration of the idle time of device $k$ just before it provides service to task $v, i t_{k, v}$, as

$$
i t_{k, v}=s(v)-\sum_{u=0}^{n}\left(s(u)+d u r_{u}\right) \cdot Y_{k}(u, v) \cdot
$$

Let function idlene $_{k}($ it) return the energy consumed by device $k$ during idle time of length it. Note that the device may be placed in a low-power state during its long idle times, as suggested, for instance, in [8][9]. For the illustration purpose, assume that device $k$ has two power states: active and sleep. Let $p_{a}$ and $p_{s}$ denote the power consumptions of the device in the active and sleep states, respectively. Let $\varepsilon_{t r}$ and $\tau_{t r}$ denote the summation of energy overheads and latency overheads associated with the two transitions into and out of the sleep state, respectively. Recall that the breakeven time is equal to $\tau_{B E}=\varepsilon_{t r} /\left(p_{a}-p_{s}\right)$. Then,

$$
\text { idlene }_{k}(\text { it })=\left\{\begin{aligned}
\varepsilon_{t r}+p_{s} \cdot i t, & \text { it } \geq \max \left(\tau_{B E}, \tau_{t r}\right) \\
p_{a} \cdot i t, & \text { otherwise }
\end{aligned}\right.
$$

Thus, the total energy consumed by the system during time period $T_{d}$ is calculated as

$$
\begin{aligned}
E_{s y s} & =\sum_{u=1}^{n} \text { ene }_{u}+\sum_{k=0}^{K} \sum_{v=1}^{n} \text { idlene }_{k}\left(Z_{k}(v) \cdot\left[1-Y_{k}(0, v)\right] \cdot i t_{k, v}\right) \\
& +\sum_{k=0}^{K} \text { idlene }_{k}\left(\text { it }_{k, n+1}+\sum_{v=1}^{n} Y_{k}(0, v) \cdot i t_{k, v}\right)
\end{aligned}
$$

Notice that when executing a periodical task set, for a device, the idle time before the first task starts and the idle time after the last task finishes actually constitute a single idle period. The third term on the right-hand side of equation (2-9) calculates the device energy consumption for such an idle period. The second term on the RHS handles all the other idle times.

The optimization problem is to minimize $E_{\text {sys }}$ with respect to constraints (2-1) to (2-8). Note that in this formulation, we ignore the energy and timing overhead associated with the voltage changes because switching of the CPU voltage normally takes between 10-100 microseconds depending on the hardware support for the DVS function. This is negligible compared to the device on/off transition times, which tend to be in the range of a few tenths of a second. The corresponding energy overhead is also small.
This problem is a nonlinear non-convex integer program over variables $s(u), x(u, i)$ and $Y_{k}(u, v)$; the worst-case computational complexity of exactly solving this problem is expected to be exponential. So we propose a three-step heuristic approach to solve the problem as follows:

1. Task Ordering: Derive a linear ordering of tasks (i.e., calculate $Y_{k}(u, v)$ values) by performing an interactive minimum-cost matching on some appropriately constructed graph (cf. section 3.)

2. Voltage Assignment: Given the task ordering implied by the schedule obtained in step 1, assign voltages and task durations (i.e., calculate $x(u, i)$ values) and exact start times (i.e., calculate $s(u)$ values) to each task so as to meet a target cycle time, $T_{d}$ (cf. section 4.)

3. Refinement: Improve the task scheduling and voltage assignment of steps 1 and 2 to increase the energy efficiency of the resulting solutions (cf. section 5.)

\section{TASK ORDERING}

In this step, we assume that the CPU voltage level is set to the maximum possible value and that the task execution times (durations) are calculated on this basis. ${ }^{3}$ The goal is to take the task graph with known task execution times and schedule it on the CPU (device 0) so as to minimize the total energy dissipation due to $\mathrm{I} / \mathrm{O}$ devices $(1, \ldots, \kappa)$ staying in the idle mode and that caused by transitioning the devices from their high-power functional state to the low-power sleep state. Notice that the summation of energy dissipation in all devices $(0, \ldots, \kappa)$ when these devices are in active states is fixed and independent of the scheduling. The scheduling only changes the duration of the idle times and the number of on to off transitions for the I/O devices.

Let tasks $_{k}$ denote the set of tasks running on device $k$ and $d e v_{u}$ denote the set of devices that are needed by task $u$. A lower bound on the total system energy dissipation, totene $_{L B}$ can be obtained by assuming that there is no energy overhead for the transitions between idle and sleep states of any device

totene $_{L B}=\sum_{u \in V} \sum_{k \in \text { dev }_{u}}$ funcpow $_{k} \cdot$ dur $_{u}+\sum_{k=0}^{\kappa}$ sleepow $_{k} \cdot\left(T_{d}-\sum_{u \in \text { task }_{k}} d u r_{u}\right)$

Now, the actual total energy includes the energy consumed by various devices when they stay in their idle modes and when they transition in and out of the sleep modes. Let's denote the schedule, $\Lambda$, by the start times of all tasks in the given task graph. Based on this information, one can linearly order the set of tasks and represent the active times of each device as a set of closed intervals. More precisely, device $k$ will be represented by a segment set, $S_{k}=\left\{s_{k, 1}, \ldots, s_{k, z}\right\} \quad(z \leq n)$ corresponding to the time intervals during which the device is in its active state.

\footnotetext{
${ }^{3}$ This is a simple heuristic used to assign task durations for this step. Other heuristic assignments are possible. Note, however, that we are only interested in the ordering of tasks after the completion of this step and will in fact calculate the exact task schedule and execution times after voltage assignment.
} 


$$
\begin{aligned}
& t_{\text {nonactive }}\left(s_{k, i}, s_{k, i+1}\right) \triangleq \begin{cases}\operatorname{start}\left(s_{k, i+1}\right)-\operatorname{end}\left(s_{k, i}\right) & \text { if } i<\left|S_{k}\right| \\
T_{d}+\operatorname{start}\left(s_{k, 1}\right)-\operatorname{end}\left(s_{k,\left|S_{k}\right|}\right) & \text { otherwise }\end{cases} \\
& F_{k, i} \triangleq F_{k}\left(s_{k, i}, s_{k, i+1}\right)= \begin{cases}1 & \text { if } t_{\text {nonactive }}\left(s_{k, i}, s_{k, i+1}\right) \geq t_{B E, k} \\
0 & \text { otherwise }\end{cases} \\
& \text { totene }(\Lambda)=\text { totene }_{L B}+ \\
& \sum_{k=1}^{\kappa} \sum_{i=1}^{S_{k} \mid-1}\left(F_{k, i} \cdot \text { transene }_{k}+\left(1-F_{k, i}\right) \cdot \text { funcpow }_{k} \cdot t_{\text {nonactive }}\left(s_{k, i}\right)\right)
\end{aligned}
$$

Here $\operatorname{start}(s)$ and $\operatorname{end}(s)$ denote the start time and end time of segment $s$ while transene $_{k}$ denotes the total transition energy cost of device $k$ to go from idle mode to the sleep mode and to return to the active mode.

Next we construct an augmented task graph (ATG) $A(V, C)$ from the given task graph $G(V, E)$ by copying $G(V, E)$ and subsequently adding/deleting some edges to/from $E$. More precisely, the new edge set, $C$, does not contain any directed edge $u v$ such that there exists another directed path from $u$ to $v$ in $C$. In addition, $C$ contains undirected edges $q r$ if tasks associated with $q$ and $r$ can be scheduled next to each other in some order. In addition, each node, $q$, in $\mathrm{V}$ (task) has three attributes: task execution time, $d u r_{q}$, task energy consumption, ene $e_{q}$, and the list of devices that are required by the task, $d e v_{q}$. Finally, each directed edge $q r$ in $C$, has an associated energy cost, extraene ${ }_{q r}$, calculated as follows

$$
\begin{aligned}
& s_{k, q} \triangleq\left[\operatorname{start}(r)-\operatorname{dur}_{q}, \operatorname{start}(r)\right] ; \quad s_{k, s u c c(r)} \triangleq\left[\operatorname{start}(r)+d u r_{r}, T_{d}\right] \\
& F_{k, q, r} \triangleq F_{k}\left(s_{k, q}, s_{k, s u c c(r)}\right) \\
& \text { extraene }_{q r}= \\
& \quad \sum_{k \in d e v_{q}-\text { dev }_{r}} \sum_{i=1}^{\left|S_{k}\right|-1}\left(F_{k, q, r} \cdot \text { transene }_{k}+\left(1-F_{k, q, r}\right) \cdot \text { funcpow }_{k} \cdot t_{\text {nonactive }}\left(s_{k, i}\right)\right)
\end{aligned}
$$

Each undirected edge between nodes $q$ and $r$ will have two such energy costs corresponding to directed edges $q r$ and $r q$. Note however that at most one of the two directed edges may be chosen as part of the scheduling solution.

The basic flow of the proposed scheduling algorithm is to iteratively find the edge with the least extra energy value and merge its two end nodes, implying that the corresponding tasks will be scheduled to run in immediate succession. For a directed edge, the ordering is fixed a priori whereas for the undirected edge, the algorithm will choose one of the two possible orderings and fix it. After each merge, the ATG is updated by removing all edges that become invalid and calculating the attributes for the newly generated node. The process continues until a single node is left in the ATG, which corresponds to a total ordering (scheduling) of all the tasks. The process continues until exactly one node remains in the modified ATG (i.e., a complete schedule is obtained.) If at any step of the algorithm, there is a tie between the extra energy costs of two candidate edges $q r$ and $u w$, then we will choose the edge that would result in the minimum total extra energy cost, extraene ${ }_{\text {tot }}$, of the resulting graph if the merge

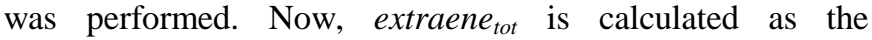
summation of the node weights of the resulting graph where the node weight is itself calculated as the average of the extra edge costs of outgoing edges from that node.
Example 1: Consider a task graph depicted in Figure 1(a). Assume that there are four devices $\{0,1,2,3\}$ with the following device utilization sets:

$$
\begin{aligned}
& \operatorname{dev}(u 1)=\{0\} \\
& \operatorname{dev}(u 2)=\operatorname{dev}(u 4)=\{0,1\} \\
& \operatorname{dev}(u 3)=\operatorname{dev}(u 5)=\operatorname{dev}(u 6)=\{0,2,3\}
\end{aligned}
$$

For the sake of simplicity, we assume that each task has a unit time duration (which is longer than its breakeven time) and that the idle power consumption of all devices is the same. In addition, each device consumes 1 unit of energy for each transition to and from the sleep state. The ATG graph of this task set is given in Figure 1(b). The directed edge $u 1 u 2$ exists in ATG, because there is a precedence constraint between nodes $u 1$ and $u 2$ and $u 2$ can be scheduled immediately after $u 1$. The presence of undirected edge $u 2 u 3$ implies that $u 2$ and $u 3$ can be scheduled next to the other without any ordering constraint. The edge labels denote the energy consumption if the start and end nodes of the edge are scheduled one after the other. For simplicity, assume all node energies are 0 .

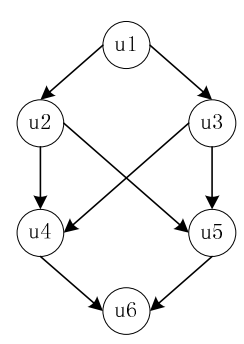

(a) Initial task graph

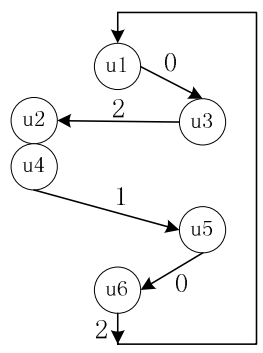

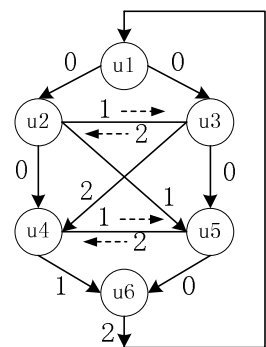

(b) ATG
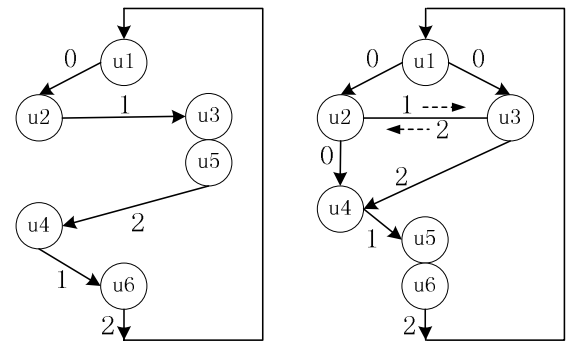

(c) ATG after merging a pair of node

Figure 1. Illustrative example for power-aware task schedule.

With this ATG, we can start the task scheduling for power management. There are five edges with minimal edge energy equal to 0 . That is, we can merge the pair of nodes associated with each of these edges without incurring additional energy cost. In Figure 1(c), three ATGs are presented, each corresponding to the merging of the node pair for one of the edges. Let us consider the left-most ATG which is generated after merging $u 2$ and $u 4$. Since there is a directed edge from $u 2$ to $u 4, u 4$ must be scheduled after $u 2$. After the $u 2-u 4$ merge, the edge from $u 3$ to $u 2$ becomes a directed edge, because originally $u 3$ had to be executed before $u 4$ which has now been merged with $u 2$ into a single node. The directed edge from $u 1$ to $u 2$ in the initial ATG disappears because after the $u 2-u 4$ merge, $u 3$ stands between $u 1$ and $u 2$ in the precedence chain.

The left-most ATG in Figure 1(c) has the minimal $E_{A T G}$ value equal to 5 . So the merge of $u 2$ and $u 4$ is selected for the first step. 


\section{Voltage Assignment}

Having generated the task schedule, we fix the ordering of tasks, but otherwise, ignore the task execution times and start times, which were heuristically set as explained at the beginning of section 3 , we can easily calculate the $Y_{k}(u, v)$ values. Thus by substituting the value of $Y_{k}(u, v)$ into the optimization problem formulated by (2-1) through (2-9), all constraints becomes linear constraints and the only unknowns become $s(u)$ and $x(u, i)$ variables. However, the optimization problem cannot be solved exactly and efficiently, because the objective function remains a non-convex function of idle times, it. We thus propose two approaches to get around this non-convexity issue.

The first one simply ignores the energy components introduced by idlene $_{k}$ in equation (2-9). The optimization problem thus becomes a linear programming problem over continuous variable $x(u, i)$, which can be solved in polynomial time. It is worth pointing out that, strictly speaking, $x(u, i)$ should take discrete values instead of continuous ones, because the number of CPU cycles executed at each operating voltage is an integer. However, when we consider a task executed in hundreds of thousands of CPU cycles, the effect introduced by rounding up to one cycle can be safely ignored.

The second approach introduces new 0-1 integer variables $W_{k}(v, h)$ to approximate the idle time $i t_{k, v}$ as follows: $W_{k}(v, h)$ $=1$ exactly if $t_{k, h} \leq i t_{k, v}<t_{k, h+1} . t_{k, h}$ and $t_{k, h+1}$ take values from a discrete set $\left\{t_{k, 1}, t_{k, 2}, \ldots, t_{k, H}\right\}$. The value of idlene $_{k}(i t)$ is approximated by

idlene $_{k}\left(i_{k, v}\right)=\sum_{h=1}^{H}$ idlene $_{k}\left(t_{k, h}\right) W_{k}(v, h)$, with $\sum_{h=1}^{H-1} W_{k}(v, h)=1$.

Consequently, constraint (2-8) is thus rewritten as

$$
\begin{aligned}
\sum_{h=1}^{H-1} t_{k, h} W_{k}(v, h) & \leq s(v)-\sum_{u=0}^{n}\left(s(u)+d u r_{u}\right) \cdot Y_{k}(u, v) . \\
& \leq \sum_{h=1}^{H-1} t_{k, h+1} W_{k}(v, h)
\end{aligned}
$$

And equation (2-9) becomes an integer linear cost function. As a result, the original optimization problem is approximated with a mixed integer linear program. The value of parameter $H$ can be adjusted to trade-off the computational complexity and the approximation accuracy.

\section{REFINEMENT}

In this section, we provide a top-level overview of an algorithm that we have developed to improve the results obtained by the first two steps. Starting from the solution obtained from steps 1 and 2, we shift the tasks together to remove redundant positive slack times. Next, we apply a greedy refinement algorithm on this solution to improve the total energy cost while meeting the timing constraint. In particular, we identify the set of critical tasks whose duration has a large impact on the system energy dissipation, e.g. a small change of the duration could enable device transitions to low power states, and change their voltage assignments accordingly. Detailed are omitted.

\section{EXPERIMENTAL RESULTS}

This experiment is conducted on a system comprising of a single CPU and three other devices. The CPU has three operating voltage/frequency levels: $1 \mathrm{~V} / 200 \mathrm{MHz}$, $1.1 \mathrm{~V} / 300 \mathrm{MHz}$ and $1.3 \mathrm{~V} / 400 \mathrm{MHz}$ [11]. The CPU and all devices support only one low-power sleep state. The power consumptions in different states, energy and timing overheads of state transitions for both the CPU and the three devices are reported in Table 1.

\section{TABLE 1}

Power and transition parameters

\begin{tabular}{ccccc}
\hline Device & $\begin{array}{c}\text { Active } \\
\text { Power }\end{array}$ & $\begin{array}{c}\text { Sleep } \\
\text { Power }\end{array}$ & $\begin{array}{c}\text { Energy } \\
\text { Overhead }\end{array}$ & $\begin{array}{c}\text { Timing } \\
\text { Overhead }\end{array}$ \\
\hline SDRAM & $0.3 \mathrm{~W}$ & $\sim 0$ & $\sim 0$ & $\sim 0$ \\
\hline HDD & $2.1 \mathrm{~W}$ & $0.85 \mathrm{~W}$ & $0.6 \mathrm{~J}$ & $400 \mathrm{~ms}$ \\
\hline WLAN & $0.7 \mathrm{~W}$ & $0.05 \mathrm{~W}$ & $0.04 \mathrm{~J}$ & $100 \mathrm{~ms}$ \\
\hline CPU & $\begin{array}{c}1.0 \mathrm{~W} \\
(200 \mathrm{MHz})\end{array}$ & $0.05 \mathrm{~W}$ & $0.3 \mathrm{~J}$ & $400 \mathrm{~ms}$ \\
& & & \\
\hline
\end{tabular}

To evaluate the effectiveness of our proposed approach, we generated five task graphs by using software package, TGFF [15], which is a randomized task graph generator widely used in the literature to evaluate the performance of scheduling algorithms. Each task graph consists of 20 to 200 tasks. All tasks require supports from SDRAM. The dependency of tasks on HDD and WLAN were randomly generated and fixed before optimization. The characteristics of different task graphs are given in the following table. For example, for task graph G1, when the CPU has its highest frequency setting, the cpu is used during $61 \%$ of the total execution whereas the SDRAM, HDD and WLAN are used for $61 \%, 29 \%$ and $42 \%$ of the total time.

\section{TABLE 2}

Characteristics of task graphs

\begin{tabular}{ccc}
\hline $\begin{array}{c}\text { Task } \\
\text { Graph }\end{array}$ & $\begin{array}{c}\text { No. of } \\
\text { Tasks }\end{array}$ & $\begin{array}{c}\text { CPU and device utilization factors at } \\
\text { max speed for the CPU } \\
\text { CPU SDRAM HDD WLAN }\end{array}$ \\
\hline G1 & 28 & 0.610 .610 .290 .42 \\
\hline G2 & 65 & 0.720 .720 .510 .39 \\
\hline G3 & 110 & 0.340 .340 .120 .23 \\
\hline G4 & 159 & 0.480 .480 .300 .25 \\
\hline G5 & 204 & 0.550 .550 .280 .36 \\
\hline
\end{tabular}

In this experiment, we compare the total system energy consumptions of the following methods:

M1: No DVS, no DPM. The CPU always operates at the highest voltage level and devices are kept active during the whole execution time. This provides the baseline compare against.

M2: DPM without any task scheduling. Tasks are executed on the CPU (which has assumed its highest frequency and voltage setting) in an un-optimized order based on their ID numbers after they become available. A method similar to the approach in [8] is used to determine the state transition sequences of all devices and the CPU.

M3: DPM with task scheduling. This method is similar to M2, except that our proposed power-aware task scheduling algorithm is used to determine the task execution sequence. 
M4: Conventional cpu-driven DVS plus DPM. Similar to M2, except that the task operating voltage is assigned to minimize the CPU power consumption. More specifically, the operating voltage setting for each task is obtained by solving the optimization problem defined in section 2 without considering the energy consumption of devices.

M5: Proposed system-aware DVS plus DPM (which have called, Power-aware Scheduling and Voltage Setting or PSVS for short.) Task scheduling and operating voltage settings are determined through the proposed three-phase framework.

TABLE 3

Normalized energy consumption results for different techniques

\begin{tabular}{ccccc}
\hline Task Graph & M2 & M3 & M4 & M5 \\
\hline G1 & 0.54 & 0.50 & 0.58 & 0.47 \\
\hline G2 & 0.67 & 0.59 & 0.63 & 0.53 \\
\hline G3 & 0.28 & 0.26 & 0.32 & 0.25 \\
\hline G4 & 0.40 & 0.35 & 0.42 & 0.34 \\
\hline G5 & 0.43 & 0.37 & 0.39 & 0.33 \\
\hline
\end{tabular}

The energy consumptions of different techniques are compared in Table 3. These values have been normalized with respect to the baseline energy consumption of M1, e.g., for G1, M2 results in total system energy consumption which is $54 \%$ of the baseline energy consumption. From this table, it is seen that compared to DPM technique without task scheduling, our proposed DPM with task scheduling can reduce energy consumption by an average of $11 \%$. Furthermore, when this method is combined with our proposed voltage assignment technique (resulting in M5 or PSVS), an additional $9 \%$ energy saving is achieved.

\section{CONCLUSIONS}

This paper addresses the problem of minimizing energy consumption of a computer system performing periodic hard real-time tasks with precedence constraints. In the proposed approach, dynamic power management and voltage scaling techniques are combined to reduce the energy consumption of the CPU and devices. The optimization problem is first formulated as an integer programming problem. Next, a threephase solution framework, which integrates power management scheduling and task voltage assignment, is proposed. Experimental results demonstrate efficiency of the proposed approach.

\section{REFERENCES}

[1] L. Benini, A. Bogliolo and G. De Micheli, "A survey of design techniques for system-level dynamic power management," IEEE Trans on VLSI, vol.8 iss.3, pp.299316, 2000.

[2] J. Luo and N. Jha, "Static and dynamic variable voltage scheduling algorithms for real-time heterogeneous distributed embedded systems," ASP-DAC, pp. 719-26, 2002.

[3] F. Gruian and K. Kuchchinski, "LEneS: task scheduling for low-energy systems using variable supply voltage processors," ASP-DAC, pp. 449-55, 2001.

[4] Y. Zhang, X. Hu, and D.Z. Chen, "Task scheduling and voltage selection for energy minimization," $D A C$, pp. 183-8, 2002.
[5] Y-H Lu, L. Benini and G. De Micheli, "Low-power task scheduling for multiple devices," CODES, pp. 39-43, 2000.

[6] V. Swaminathan and K. Chakrabarty, "Pruning-based energy-optimal device scheduling for hard real-time systems," CODES, pp.175-80, 2002.

[7] Y-H Lu, L. Benini and G. De Micheli, "Power-aware operating systems for interactive systems," IEEE Trans. on VLSI, vol.10 iss.2, pp. 119-34, 2002.

[8] V. Swaminathan and K. Chakrabarty, "Energy-conscious, deterministic I/O device scheduling in hard real-time systems," IEEE Trans. on CAD, vol.22 iss.7, pp.847-58, 2003.

[9] J. Liu and P.H. Chou, "Optimizing mode transition sequences in idle intervals for component-level and system-level energy minimization," ICCAD, pp. 21-28, 2004.

[10] R. Jejurikar and R. Gupta, "Dynamic voltage scaling for system-wide energy minimization in real-time embedded systems," ISLPED, pp. 78-81, 2001.

[11] K. Choi, W. Lee, R. Soma and M. Pedram, "Dynamic voltage and frequency scaling under a precise energy model considering variable and fixed components of the system power dissipation," ICCAD, pp. 29-34, 2004.

[12] Q. Qiu and M. Pedram, "Dynamic power management based on continuous-time Markov decision processes," DAC, pp. 555-561, 1999.

[13] T. Simunic, L. Benini, A. Acquaviva, P. Glynn and G. De Micheli, "Dynamic voltage scaling and power management for portable systems," DAC, pp.524-529, 2001.

[14] S. Irani, S. Shukla and R. Gupta, "Algorithms for power savings," SODA, pp. 37 - 46, 2003.

[15] http://ziyang.ece.northwestern.edu/tgff. 